\title{
Factores que afectan a la credibilidad en el sistema sanitario occidental y las decisiones en el tratamiento de la salud. Una revisión de la literatura
}

\section{Factors that affect credibility in the western health system and decisions in the treatment of health. A review of literature}

\author{
Blanca María Muñoz Ortína
}

a Departamento de Periodismo y Comunicación Audiviosual, Universidad Carlos III de Madrid, España

\begin{abstract}
Resumen
Introducción: Algunos autores apuntan a que se observa un aumento de la desconfianza en el sistema sanitario. Objetivo: Este trabajo pretende analizar algunos de los factores que pueden estar afectando a la confianza en el sistema sanitario occidental y a la toma de decisiones de las personas, reflexionar en base a ello, y sobre cómo reforzar la confianza de los ciudadanos en la medicina convencional. Método: Se realiza una revisión de la literatura científica y no científica relacionados con la temática del trabajo haciendo uso de tres estrategias de búsqueda: 1) Motores de búsqueda (Google); 2) un periódico nacional en su versión digital (el País) y un periódico digital (Diario Médico), elegidos aleatoriamente; 3 ) gestores bibliográficos de literatura científica como Pubmed y Web of Science. Resultados: Los medios de comunicación, Internet y redes sociales, y movimientos anticiencia son los ingredientes perfectos para la generación de desconfianza en el sistema sanitario y manipular las decisiones de las personas para el tratamiento de la salud y la enfermedad. Conclusiones: reforzar de nuevo la confianza de la ciudadanía en un sistema sanitario efectivo requiere del trabajo conjunto de varios colectivos.
\end{abstract}

Palabras clave: sistema sanitario occidental; medicina alternativa y complementaria; desconfianza; Internet; medios de comunicación; movimientos anticiencia.

\begin{abstract}
Introduction: Some authors suggest that there is an increase in distrust in the health system. Objective: This work aims to analyze some of the factors that may be affecting the trust in the western health system and the decision-making of people, reflect about this, and how to strengthen the confidence of citizens in conventional medicine. Method: A review of the scientific literature and non-indexed articles related to the subject of work is carried out using three search strategies: 1) web search engines (Google); 2) a national newspaper in its digital version (el País) and a digital newspaper (Diario Médico) randomly chosen; 3) bibliographic managers of scientific literature such as Pubmed and Web of Science. Results: The media, Internet and social networks, and anti-science movements are the perfect ingredients for generating distrust in the health system and manipulating the decisions of people for the treatment of health and disease. Conclusions: Reinforcing citizens' trust in an effective health system requires the joint work of several groups.
\end{abstract}

Key words: Western health system; distrust; alternative and complementary medicine; Internet; media; anti-science movements. 


\section{Introducción}

$\mathrm{L}$ a ciencia moderna estableció el método científico como forma de adquirir conocimiento. Se trata de un conjunto de pasos ordenados que permiten comprobar una hipótesis de partida, de tal manera que lo que se percibe sobre un hecho esté libre de interpretaciones humanas y por tanto de toda subjetividad. Está basado en la experimentación cuyos patrones, fenómenos u objetos deben poder ser medibles y apoyarse en los pilares básicos de la reproducibilidad y la refutabilidad o falsabilidad. Es decir, los resultados del experimento deben poder reproducirse por otros y poder ser contrastados y refutados con otras hipótesis o teorías.

La pseudociencia o falsa ciencia se basa en afirmaciones de hipótesis o creencias que no siguen un procedimiento de verificación válido con el que se pueda comprobar su fiabilidad y su falsabilidad por lo que no cumple ninguna de las premisas que se le imponen al método científico para la adquisición de conocimiento. En otras palabras, la pseudociencia está sujeta a la percepción de la persona, a la experiencia individual y a la subjetividad. Es por este motivo por lo que no puede considerarse como una ciencia ya que el objeto de estudio, la hipótesis, no puede ser sometida a un análisis riguroso. Además, la información que se ofrece es imprecisa por lo que no puede comprobarse su efectividad.

En el ámbito de la salud, la Medicina Tradicional y Complementaria (MTC) recoge numerosas técnicas, productos y tecnologías que carecen del fundamento científico requerido para que pueda ser considerado como tal, es decir, según hemos explicado en el párrafo anterior, la MTC queda acogida dentro del marco de lo que conocemos como una pseudociencia. La OMS (2013) integra dentro de la medicina tradicional, la homeopatía, la acupuntura, la medicina china, la naturoterapia, la fitoterapia, los tratamientos con suplementos nutricionales y vitaminas, la reflexología, la quiropraxia, el yoga, el reiki, y un largo etcétera. Estas técnicas son, sin embargo, integradas y aceptadas por la comunidad médica (Coulter, 2004) y por el grueso de la ciudadanía.

Actualmente nos encontramos inmersos en una batalla (casi literal) por demostrar quién dice la verdad, científicos versus pseudocientíficos; a quién debe creer el ciudadano de a pie cuyo conocimiento, en muchos casos, se basa en una fe ciega hacia los medios de comunicación, y también hacia lo que dice la vecina del cuarto piso. Porque, por ejemplo, una mala experiencia en lo que respecta al trato que recibe una persona por parte del personal médico o sanitario, o el fracaso de un tratamiento médico, puede ser crucial para desacreditar al sistema que aplica.
En este sentido, existen estudios que revelan la pérdida de confianza del paciente en la salud pública occidental y para explicar el motivo por el que esto está sucediendo, Mohammed Siahpush establece tres hipótesis. La pérdida de confianza en la medicina pública occidental se debe a que 1) existe una insatisfacción con el sistema de salud público; 2) existe una insatisfacción con el trato que los pacientes reciben por parte del personal sanitario, 3) la CAM suponen una filosofía de vida más congruente con la recuperación de un sistema de valores tradicionales (M, 1998). Otros autores indican además, que este comportamiento coincide con el aumento del uso de la medicina complementaria y alternativa (CAM); la popularización de estas técnicas se debe a que la medicina convencional no cubre ciertas necesidades de los pacientes. Las personas no están de acuerdo con los métodos que utiliza la medicina convencional por lo que van a buscar otras maneras de atender a su salud. Muchas aseguran que la CAM proporciona un entorno terapéutico más positivo, más humano y empático. En este sentido, sus justificaciones se alinean sobre el déficit de la medicina occidental en lo referente al trato humano, para dar un sistema de atención de la salud eficaz y satisfactorio (Nadia Corp , J. L. Jordan, 2018; Salamonsen \& Ahlzén, 2018).

Ante el incremento del uso de estas terapias para el tratamiento de enfermedades como el cáncer, grupos de científicos, colectivos médicos, y estudiantes han comenzado a lanzar manifiestos y peticiones de firmas dirigidas a los medios de comunicación, a las agencias reguladoras, a los colegios de profesionales, y a los organismos públicos, para protestar contra la proliferación de este tipo de terapias pseudocientíficas que se apoyan en argumentos propios de la charlatanería y que en algunas ocasiones están amparadas por instituciones públicas y empresas médicas profesionales. Los galenos aseguran que lo malo no es tener creencias, que es en lo que se basan las pseudociencias, sino que la vendan como algo demostrado científicamente y que además aseguren que pueden curar enfermedades como el cáncer. Este hecho, aseguran, es el que incita a las personas a abandonar sus tratamientos basados en la evidencia científica, y lo sustituyen por fraudes que llegan a costarles la vida

\section{Objetivos del trabajo}

El objetivo de este trabajo se dirige a analizar algunos de los factores que pueden estar afectando a la confianza en la medicina convencional y a las decisiones de las personas para tratar su salud. En base a ello se pretende discutir y reflexionar sobre cómo se podría reforzar la confianza de los ciudadanos en la medicina convencional. 


\section{Materiales y métodos}

Este trabajo es una revisión bibliográfica y de la información que circula en internet sobre el tema del trabajo. En total se han analizado 8 artículos en español; 3 en inglés; 1 tesis doctoral; 1 libro; 2 entradas de blogs diferentes (ciencia mundana y La botica escondida); 3 noticias de dos periódicos. Para realizar el estudio se han empleado tres estrategias de búsqueda orientadas siempre hacia la temática: 1) motores de búsqueda de Google; 2) un periódico nacional en su versión digital (el País) y un periódico digital (Diario Médico) elegidos al azar; 3 ) dos gestores bibliográficos de literatura científica: Pubmed y Web of Science. Las palabras clave para la recopilación de la información han sido "medicina alternativa y complementaria", "bulos en la salud", "medios de comunicación y pseudociencias" (para las estrategias 1 y 2); "pseudoscience", "pseudotherapy" (para la estrategia 3). Los criterios de elegibilidad de artículos han sido los siguientes: artículos relacionados con la temática; que hayan sido publicados entre los años 2000 y 2018 (solo hay una excepción de un artículo de 1998, del autor Mohammed Siahpush. Se ha incluido por su pertinencia y relevancia de lo que se pretende analizar); en inglés y español.

\section{Resultados}

Se introduce en este apartado los factores que se han analizado y que se cree, pueden estar afectando a la confianza del ciudadano en la medicina convencional y las decisiones de las personas en el tratamiento de su salud, esto es, los medios de comunicación, Internet y redes sociales y los movimientos anticientíficos.

\section{Medios de comunicación}

De Azcarraga señala la falta de prudencia a la hora de reproducir cierto tipo de noticias. Afirma por un lado que "la reproducción de noticias que otros afirman es una caja de resonancia hacia la superchería" y por otro denuncia la existencia de una "falta de contrapeso crítico por parte de los medios de comunicación". Y es que el problema reside en que los media muchas veces "supervaloran la capacidad crítica de los lectores" (De Azcarraga, 2015), y "cuando las pseudociencias se difunden con total normalidad y sin crítica alguna en los medios de comunicación, se institucionalizan y, por extensión, adquieren legitimidad a partir de la credibilidad supuesta a los medios de comunicación (López Cantos \& Millán Yeste, 2018). A menudo programas de televisión ${ }^{1}$ y radio ${ }^{2}$ que son emitidos en horarios de máxima audiencia son denunciados por expertos debido a su contenido pseudocientífico. Este problema, según Valerio-Matas \& Muñoz Sandoval, subyace a la falta de conocimiento científico por parte de quien dirige estos medios de comunicación (Valero-Matas \& Muñoz Sandoval, 2017).

\section{Movimientos anticiencia}

Nespereira-García (2015), en su estudio sobre el uso de la retórica como herramienta para la gestión y la comunicación del riesgo sanitario, apunta a que en la actualidad las personas son más reflexivas con respecto a la salud y el riesgo pero existen diferencias cognitivas en los diversos estratos sociales, en cuanto a la manera de percibir un riesgo sanitario. Las tensiones entre el contexto sociocultural y el conocimiento y el razonamiento científico se hacen evidentes en las sociedades occidentales ante un escenario en el que el rechazo al seguimiento de los protocolos de vacunación infantil se han incrementado desde 2011, cuando comienza a registrarse una mayor incidencia de enfermedades infecciosas como el sarampión o la rubeola, en España y otros países (ibídem). Según Valero-Matas \& Muñoz Sandoval (2017), "existe un miedo social a la ciencia difundido por intelectuales" que genera desconfianza hacia las autoridades cientificas. Y, por si fuera poco, el rigor que se le atribuye al método científico como forma objetiva de adquirir conocimiento se pone en tela de juicio cuando se hacen evidentes el fraude científico, los engaños corporativos, y la mala praxis por parte de los grandes lobbies de las farmacéuticas: conductas que, además de no favorecer el argumento científico, promueven la desconfianza hacia las autoridades científicas, generan rechazo y favorecen el ataque dogmático contra la ciencia, incrementando la incertidumbre y los pensamientos anticientificos (ibídem).

Diferentes grupos pseudocientíficos liderados por intelectuales apoyan sus críticas contra la ciencia resaltando sus aspectos negativos y sus limitaciones, y construyen sus argumentos vinculados estrechamente con el poder (ibídem). Para Jesús García Blanca (2017) "el Modelo

\footnotetext{
Programas de Televisión española como «Saber Vivir»

https://elpais.com/cultura/2018/08/21/television/1534861300_084559. html y el programa de Javier Cárdenas https://elpais.com/elpais/2017/12/05/ ciencia/1512465479_981726.html han ofrecido hasta hace poco contenido pseudocientífico durante las horas de mayor audiencia.

2 El Congreso de los Diputados emitió una queja por el contenido pseudocientífico del programa de radio «En Cuerpo y Alma» http://www.congreso.es/12p/e1/ e_0019125_n_000.pdf
} 
Médico Hegemónico se consolida durante el siglo XX, favorecido por la reconversión de las multinacionales farmacéuticas, especialmente tras la Segunda Guerra Mundial, transformándose en una poderosa herramienta de poder que controla la formación, la información, la investigación y los servicios sanitarios progresivamente dominados por la súper especialización y la tecnología", para Costa Vercher ${ }^{3}$ (2016) la teoría de la infección es completamente errónea porque deriva de una invención de la industria y del sistema de imponer un tipo de consumo tóxico, totalmente irracional, y totalmente inútil. Además, comenta que las vacunas no tienen ningún sentido biológico. Estos autores y muchos otros, apoyan sus argumentos y elaboran sus discursos en base a las teorías de otros pensadores como Feyerabend o Huxley, para quienes la ciencia y la tecnología son herramientas de dominación y control social (Valero-Matas \& Muñoz Sandoval, 2017) o Foucault, quien establece una serie de silogismos que desembocan en su afirmación sobre que "la ciencia es un arma política porque implica saber, y el saber es el pilar del poder (...) la dirección del saber será siempre hacia unos pocos privilegiados mientras que para el resto el lenguaje científico es intimidatorio, incomprensible e inaccesible". En definitiva, para Foucault el discurso de 'la verdad' se justifica para "aplastar y dominar, para exigir conformidad y sumisión" (Ovejero Bernal \& Pastor Martín, 2001).

\section{Internet y Redes Sociales}

Ante el nuevo paradigma de la comunicación que ofrece Internet, Barbara Yuste afirma que la web 2.0 ha conseguido consolidar un modelo de comunicación en el que el proceso comunicacional adquiere un sentido bidireccional. Yuste analiza en su estudio cómo las redes sociales figuran como la herramienta más consultada por los jóvenes para mantenerse informados (Yuste, 2015). Nespereira-García, no duda en cargar de cierta responsabilidad al hecho de que este nuevo paradigma comunicacional está promoviendo un aumento en la tendencia al rechazo y la desconfianza hacia las autoridades cientificas (Nespereira-García, 2015). Y es que Internet favorece la popularización de productos y terapias "milagro", sanadoras de cáncer como el MMS (vendido como The Miracle Mineral Solution) del que aseguran tener propiedades sanadoras frente a parásitos, el VIH o el cáncer. Otras personas publican en sus blogs curas milagrosas para el cáncer (Valero-Matas \& Muñoz Sandoval, 2017). En Internet y las redes sociales circula una pandemia de noticias falsas que implican principalmente al tema de las pseudoterapias4.

\footnotetext{
${ }^{3}$ Entrevista extraída del blog «laboticaescondida.blogspot.com» al Doctor Enrique Costa Vercher (2016) https://laboticaescondida.blogspot.com/2016/04/vacunas-unareflexion-critica.html

${ }^{4}$ I Estudio de bulos en la salud, presentado en el I Congreso Salud sin Bulos celebrado
}

\section{Discusión}

En la Antigüedad eran los monjes quienes reproducían los textos manuscritos y el feudalismo les otorgaba el derecho para ejercer la agenda setting y manipular al pueblo. Séis siglos más tarde de que Johannes Gutenberg inventara la imprenta, la tecnología que causara un importante cambio de paradigma socio-económico y revolucionara la organización social del siglo XV, han llegado Internet y las redes sociales originando un efecto similar, aunque sin duda, a una velocidad mucho mayor.

La revolución digital en la que nos encontramos inmersos ofrece al internauta un inmenso océano de información, pero, como apunta Nicholas Carr en su libro Superficiales ¿Qué está haciendo Internet con nuestras mentes?, sin embargo, el escaso tiempo que éste posee, deja poco espacio para la profundización y la reflexión. Podemos decir que, la sociedad está siendo víctima de una infoxicación que llama a la superficialidad del tratamiento de la información donde "la multitarea, instigada por el uso de Internet, nos aleja de formas de pensamiento que requieren reflexión y contemplación, nos convierte en seres más eficientes procesando información, pero menos capaces para profundizar en esa información, y al hacerlo no solo nos deshumanizan un poco sino que nos uniformizan" (Carr, 2010).

Esta uniformización a la que se refiere Carr, debido a esa falta de reflexión, o quizá también de formación, en vista a la gran cantidad de bulos sobre la salud que circulan por las redes, ha llevado a la determinación de algunas personas, médicos e investigadores de la salud, a crear plataformas en Internet con el fin de informar y ayudar a los ciudadanos a desmontar e identificar las noticias falsas. Una de ellas, Salud Sin Bulos ${ }^{5}$ advierte en su página que estos bulos, o noticias falsas, suelen ser "temas relacionados con la alimentación y la nutrición, dietas milagro o prevención y tratamiento de enfermedades como el cáncer los más habituales de encontrar entre los mensajes que nos llegan por Facebook, WhatsApp o Twitter".

En lo referente a los medios de comunicación, el ciudadano confía en ellos. El problema sobreviene cuando éstos presentan las pseudociencias como un instrumento de valor a consultar (De Azcarraga, 2015). Este hecho, junto con la gran cantidad de literatura sobre teorías de

\footnotetext{
en el Hospital de La Princesa de Madrid el mes de noviembre 2018 https://www. diariomedico.com/salud/siete-de-cada-diez-medicos-atienden-pacientes-influidos-porbulos-sobre-salud.html

${ }^{5}$ Salud Sin Bulos es una iniciativa de la Asociación de Investigadores en eSalud (AIES) puesta en marcha para combatir los bulos de salud en Internet y contribuir a que exista información veraz y contrastada sobre salud en la red. Para visitar la plataforma https://saludsinbulos.com/. Otras plataformas son Apetp, OMC, StopPseudociencias 0 Enfermería Sin Pseudociencias.
} 
la conspiración contra la salud del pueblo, contribuye a aumentar la complejidad de la realidad y la incertidumbre a un público que "prefiere creer en una falsedad simple, que no obligue a razonar, a estudiar una verdad complicada" (ibídem).

No obstante, no es que la pseudociencia sea mala por el hecho de no ajustarse a la evidencia científica, por ejemplo el yoga está clasificado por la OMS (2013) como una falsa ciencia, y sin embargo el número de estudios que se inclinan a favor de los beneficios que se obtienen tras su realización, está experimentando un crecimiento positivo (Lefno, 2015). Es la charlatanería ${ }^{6}$ entendida ésta en su acepción de "embaucador que persigue la venta, muchas veces fraudulenta, de algún tipo de producto, remedio, elixir, ideología, etc." con sus discursos persuasivos, construidos en diferentes tipos de pensamiento: el pesimista, que niega el conocimiento objetivo y la racionalidad; el pensamiento postmoderno cuyo discurso adquiere la forma totalmente subjetiva; los detractores epistemológicos que se oponen a los procedimientos desarrollados para alcanzar los objetivos; o los fundamentalistas que toman la ciencia como un dogma, y los escépticos niegan la evidencia científica (Valero-Matas \& Muñoz Sandoval, 2017). Otras personas se limitan a la tergiversación ${ }^{7}$ de conceptos biológicos como Jesús García Blanca en su libro de "el rapto de Higea" donde explica erróneamente que las mitocondrias son bacterias. También se dedica a sembrar la duda y el miedo, asegurando que el sistema inmunitario no existe, o que el SIDA es una invención, entre otras más.

Pese a todo lo planteado en este trabajo, este estudio se encuentra condicionado por las limitaciones de tiempo. Las ideas que aquí se exponen no son en absoluto determinantes por lo que una revisión más exhaustiva de la literatura científica y de la literatura gris podría ayudar en futuras investigaciones a establecer otros ángulos de visión para afrontar el problema planteado.

\section{Conclusiones}

"Cuando una mentira se repite mil veces se convierte en verdad". Es una frase atribuida a J.Goebbels. Algo parecido está ocurriendo en la era de la información, salvo que en esta ocasión las noticias falsas corren como la pólvora en las redes sociales y no es necesario que se repitan tanto para que muchas personas se las crean. Controlar las fake news para evitar la manipulación requiere de un esfuerzo de todos los colectivos sociales, tanto de

\footnotetext{
${ }^{6}$ Definición extraída de https://es.wikipedia.org/wiki/Charlatán

7 Blog de ciencia mundana. Creado por un ex investigador científico, es un blog dedicado a la crítica sobre la mala ciencia y a la pseudociencia https:/ cienciamundana.wordpress.com/2017/10/02/jesus-garcia-blanca-y-el-rapto-de-higea/
}

los servicios de información y comunicación, como de las autoridades sanitarias y científicas, y de los ciudadanos. Identificar y denunciar este tipo de actos es crucial para no perpetuar la mentira, sobre todo en temas tan importantes como la salud.

No se trata sin embargo de imponer o censurar la realización de prácticas que no están sujetas a una evidencia científica sino más bien de incentivar a las personas a tener un pensamiento más crítico con respecto a la información que reciben. En este sentido urge la implantación y el desarrollo de políticas científicas que acerquen la ciencia a los ciudadanos y les ayuden en el discernir sobre qué información es verídica y cuál no lo es e identificar cuándo se trata de charlatanería.

En relación a esto último cabe hacer mayor hincapié en la educación, no solo de niños sino de todos los colectivos susceptibles de caer en el engaño. Es decir, de aquellos que carecen de formación científica. Endurecer el castigo ante el fraude científico y el engaño deliberativo y establecer políticas científicas que regulen la praxis honesta por parte de cualquier organismo dedicado a la investigación científica. En el campo de la comunicación, no confundir a las personas sobre lo que es cada cosa o sobre sus efectos, y para ello es necesario hacer más periodismo científico de calidad y dejar que sean éstos quienes se encarguen de elaborar la información que sea de su competencia.

\section{Referencias Bibliográfica}

Blanca, J. G. (2017). La medicalización de las madres como distorsión mecanicista de los comienzos de la vida humana. Daimon. Revista Internacional de Filosofía, 141-147. https://doi.org/dx.doi.org/10.6018/ daimon/302601

Carr, N. (2010). Superficiales. ¿Qué está haciendo Internet con nuestras mentes? Bogotá Colombia: Taurus.

Coulter, I. (2004). Integration and paradigm clash: The practical difficulties of integrative medicine. The Mainstreaming of Complementary and Alternative Medicine, 103-122. Retrieved from https://www.rand. org/pubs/external_publications/EP20040002.html

De Azcarraga, J. A. (2015). Los medios de comunicación frente a las pseudociencias, 38-49.

Lefno, A. L. (2015). Tesis Doctoral. Las Paradojas del Yoga Candidata Andrea Lizama Lefno, 1-350. Retrieved from https://www.tdx.cat/bitstream/ handle/10803/298326/all1de1.pdf;sequence=1 
López Cantos, F., \& Millán Yeste, J. (2018). La difusión de discursos pseudocientíficos en la radio pública española. El programa Complementarios de RNE-Radio 5. Revista Latina de Comunicacion Social, (73), 317-330. https:// doi.org/10.4185/RLCS-2018-1257

M, S. (1998). Postmodern values, dissatisfaction with conventional medicine and popularity of alternative therapies. Journal of Sociology, 34(1), 58-70.

Nadia Corp , J. L. Jordan, P. R. C. (2018). Justifications for using complementary and alternative medicine reported by persons with musculoskeletal conditions: A narrative literature synthesis. https://doi.org/https://doi. org/10.1371/journal.pone.0200879

Nespereira-García, J. (2015). La retórica como herramienta para la gestión y la comunicación del riesgo sanitario. Revista Española de Comunicación en Salud, 6(2), 222-233. Retrieved from http://e-revistas. uc3m.es/index.php/RECS/article/view/2941

OMS. (2013). Estrategia de la OMS sobre medicina tradicional 2014-2023. Organización Mundial de La Salud, 72. https://doi.org/10.1212/01. wnl.0000282763.29778.59

OvejeroBernal,A., \&Pastor Martín, J. (2001). La dialéctica saber/poder en Michel Foucault: un instrumento de reflexión crítica sobre la escuela. Aula Abierta, Universidad de Oviedo, (77), 99-110. Retrieved from http://dialnet.unirioja.es/servlet/articulo?codigo $=45498$

Salamonsen, A., \& Ahlzén, R. (2018). Epistemological challenges in contemporary Western healthcare systems exemplified by people's widespread use of complementary and alternative medicine. Health, 22(4), 356-371. https:// doi.org/10.1177/1363459317693408

Valero-Matas, J. A., \& Muñoz Sandoval, C. A. (2017). Las Pseudociencias Como Problema Social En La Era Tecnocientífica. Un Recorrido Por La Ciencia Y Sus Enemigos Dentro Y Fuera. Aposta. Revista de Ciencias Sociales, (75), 8-34. Retrieved from http://ezproxybib. pucp.edu.pe:2048/login?url=http://search.ebscohost. com/login.aspx?direct=true \&db=fua \&AN=125528504\&la $\mathrm{ng}=\mathrm{es} \&$ site=eds-live\&scope=site

Yuste, B. (2015). Las nuevas formas de consumir información de los jóvenes. Revista de Estudios de Juventud, 108, 179-191. Retrieved from http://goo.gl/ eqg9UF 